EXTENDED REPORT

\title{
Short wavelength automated perimetry and tamoxifen use
}

\section{A Eisner, D F Austin, J R Samples}

See end of article for authors' affiliations

.....................

Correspondence to: Alvin Eisner, PhD, Neurological Sciences Institute, Oregon Health and Science University, West Campus, $505 \mathrm{NW}$ 185th Ave, Beaverton, OR 97006, USA; eisnera@ ohsu.edu

Accepted for publication 19 April 2003

\begin{abstract}
Background/aims: Oestrogen receptors (ORs) have been reported to be present in the retina, and the selective oestrogen receptor modulator tamoxifen has been reported to affect colour vision. This study aimed, therefore, to determine whether standard doses of tamoxifen affect visual sensitivities mediated via short wavelength sensitive (SWS) cones.

Methods: Two types of visual fields were measured for middle aged women who were being treated with $20 \mathrm{mg}$ of tamoxifen daily as adjuvant therapy for early stage breast cancer. Visual fields were measured using short wavelength automated perimetry (SWAP) and frequency doubling perimetry (FDP). For SWAP, 24-2 visual fields were analysed. No subjects had tamoxifen retinopathy or other eye disease. For each type of visual field, mean deviations (MDs) were assessed as a function of the duration of tamoxifen use, using a cross sectional design. In addition, the difference between the two types of MDs was computed after standardisation of each type of MD separately, and this difference itself was evaluated as a function of the duration of tamoxifen use. Duration dependent changes for SWAP were further evaluated as a function of eccentricity within the visual field, and the visual field data were compared with foveal data obtained psychophysically.

Results: SWAP sensitivities depended on the duration of tamoxifen use. Subjects who used tamoxifen for about 2 years or less were significantly more likely than subjects who had longer use to have high MDs. The difference between the standardised SWAP and FDP MDs likewise was significantly related to the duration of use, whereas duration of use effects for FDP itself were reduced or absent. Although the duration of use effect observed for SWAP was strongest in the peripheral portion of the visual field, there was evidence of changes in SWS cone mediated vision even at the fovea.

Conclusion: Standard dosages of tamoxifen can affect SWAP visual fields. The effects of tamoxifen are not equivalent for SWAP and FDP, indicating that tamoxifen affects some types of visual pathways preferentially or selectively. SWS cone pathways, in particular, are affected. SWAP appears able to reveal effects of tamoxifen occurring years before completion of the standard 5 year regimen of use.
\end{abstract}

$\mathrm{S}$ lective oestrogen (estrogen) receptor modulators (SORMS, SERMS) are substances that act as oestrogen receptor (OR) agonists in some tissues and as OR antagonists in other tissues. ${ }^{12}$ Tamoxifen is a SORM that acts as an OR antagonist in breast tissue and, therefore, is used as adjuvant therapy against breast cancer by women who have had tumours positive for ORs. ${ }^{3}{ }^{4}$

Because the retina contains ORs, ${ }^{5-8}$ tamoxifen has the potential to affect vision. Indeed, standard doses of tamoxifen have been shown to reduce colour discrimination, apart from any observable effects on the lens, cornea, or macula. ${ }^{9}$ Although tamoxifen has been reported not to alter visual thresholds obtained using "white on white" automated perimetry (Humphrey 10-2), ${ }^{9}$ it might be expected to alter visual thresholds obtained using short wavelength automated perimetry (SWAP). This is because the "blue on yellow" test on background stimulus combination used for SWAP isolates the threshold responses of short wavelength sensitive (SWS) cone pathways, ${ }^{10}{ }^{11}$ which mainly subserve colour vision. ${ }^{12}$ When the threshold response properties of these pathways are assessed using "blue on yellow" stimulus combinations, there appears to be more age related change for women than for men, at least foveally. ${ }^{13-15}$ For instance, the colour appearance of stimuli detected via SWS cones is more likely to become desaturated (whiter) with age for women. ${ }^{13} 15$ If the changes among women stem from alteration of oestrogen levels or of OR function, ${ }^{16}$ SORMS may affect response properties of SWS cone pathways. However, the effects of SORMS on SWS cone mediated visual response have not been studied. This study used SWAP and foveal psychophysics to test the hypothesis that tamoxifen affects visual sensitivity mediated via SWS cones. Visual fields were also obtained using frequency doubling perimetry (FDP), which taps the visual pathways that mediate detection of flicker. ${ }^{17}$

Tamoxifen typically is used as adjuvant therapy for 5 years, ${ }^{18}$ for two reasons. Firstly, tamoxifen is thought to lose utility as an OR antagonist in breast tissue by 5 years duration of use. ${ }^{19}$ Secondly, by acting as an OR agonist in uterine tissue, tamoxifen increases the risk of endometrial cancer. ${ }^{20}$ Because the ability of tamoxifen to affect ORs in the breast may change with time, its ability to affect SWS cone mediated vision likewise may change with time. The present study used a cross sectional design to evaluate effects of tamoxifen as a function of duration of use.

Although this study was designed to evaluate effects of tamoxifen specifically, the distribution of sensitivities made it possible to discern individual factors expected to act as determinants of SWAP visual fields more generally. ${ }^{21}$ These determinants were assessed by relating SWS cone mediated sensitivities from the fovea and central portions of the visual field to SWAP sensitivities in the periphery. A multilinear regression model of peripheral SWAP sensitivities is presented in the second part of the Results section.

\section{PATIENTS AND METHODS Subjects}

The study population consisted of 47 women ages 4269 years, who were using $20 \mathrm{mg}$ of tamoxifen daily as adjuvant therapy for OR positive breast cancer. Their average age was 55.2 years (SD 6.1 years). 
All subjects met stringent eligibility criteria, which included 20/20 or better acuity in the eye used for foveal psychophysical testing, and $20 / 25$ or better acuity in the fellow eye. In addition, all subjects had intraocular pressures (IOP) $\leqslant 22 \mathrm{~mm} \mathrm{Hg}$ in each eye (Goldmann applanation), $\leqslant 2 \mathrm{~mm} \mathrm{Hg}$ IOP difference between the two eyes, $\leqslant 5$ dioptres myopia in each eye, and normal colour vision (denoted by acceptance of a normal Rayleigh match ${ }^{22}$ and no worse than one minor transposition error per eye on the D-15 test of colour discrimination under Macbeth illumination ${ }^{23}$ ). None of the subjects had a history of eye disease, diabetes, ocular surgery, or ocular hypertension, and none used any medications (except tamoxifen ${ }^{9}$ ) known to affect vision. Women were excluded from the study if, after testing, they were determined on masked evaluation of stereoscopic colour optic nerve head and fundus photographs to have pathological or suspicious ocular changes. Every subject was fully active and able to carry on all precancer activities without restriction. Every subject had completed primary treatment for breast cancer.

Subjects were recruited mainly via notices in campus publications, women's health clinics, and breast cancer survivor email message boards. All subjects were unpaid volunteers. This study was approved by the OHSU institutional review board and by the OHSU Cancer Institute. Written informed consent was obtained after explanation of the nature and possible consequences of the study.

\section{Overview of procedures}

The procedures included preliminary tests of visual function and eye health to ascertain eligibility and to determine optical correction; psychophysical tests of visual function to assess SWS cone mediated response at the fovea; SWAP and FDP visual field tests to assess peripheral vision; and fundus and optic nerve head photography to further ascertain eligibility. The psychophysical stimulus parameters were changed part way through the study in order to include yellow background illuminances that were $\operatorname{dim}$ enough to preclude much desensitisation of SWS cone mediated response. In addition, 30-2 SWAP visual fields were replaced with 24-2 SWAP visual fields. Thirty four subjects were tested using the revised set of psychophysical parameters. These are the only psychophysical data reported in this paper. Of the 34 subjects, 23 were newly enrolled and 11 had been tested using the original set of parameters.

\section{Preliminary testing}

Visual acuity was assessed using a Humphrey Instruments model 599 autorefractor (Zeiss Humphrey, Dublin, CA, USA). Several subjects could not be autorefracted to meet eligibility criteria but, instead, used corrective lenses to meet eligibility criteria. In these cases, acuity was assessed with a Snellen eye chart. When the acuity of the two eyes differed the eye with the better acuity was selected for foveal psychophysical testing. When there was no difference the subject's preferred eye was selected. In the remaining cases, the eye with the lesser spherically equivalent correction was selected. An ophthalmologist (usually JRS) measured IOP and examined the anterior and posterior portions of both eyes using slit lamp biomicroscopy and undilated direct ophthalmoscopy, respectively. The D-15 test was administered for each eye separately, and a forced choice anomaloscope screening test ${ }^{22}$ was administered for one eye.

\section{Foveal psychophysical testing battery}

This battery consisted of a series of increment threshold measurements for various test on background stimulus combinations. All measurements were made using a Maxwellian view apparatus, described previously. ${ }^{24}$ Its main features include (a) a chin rest and rubber eyepiece to facilitate proper subject alignment, (b) a small exit pupil $(1.21 \mathrm{~mm})$ to ensure that subjects' natural pupils do not affect retinal light levels, and (c) freedom to adjust field stops along the optical axis to correct for spherical refractive error.

SWS cone mediated thresholds were measured at each of three $580 \mathrm{~nm}$ background illuminance levels: 2.0, 1.6, and 3.6 $\log \mathrm{td}$, in this order. Test stimuli were $440 \mathrm{~nm}, 3^{\circ}$ diameter discs centred on the background and modulated at $1.5 \mathrm{~Hz}$. Additional test wavelengths were used to verify isolation of SWS cone mediated response at $440 \mathrm{~nm} .{ }^{15}$ Fixation was aided by crosshairs with a $4^{\circ}$ central gap. Steady state thresholds were measured after subjects viewed the background for at least 3 minutes ( 2 minutes for the $1.6 \log$ td background). Thresholds were obtained using an ascending method of limits. The step size was $0.06 \log$ units, and threshold was computed from the average of four settings. When thresholds were measured dynamically as a function of time after onset of a background, the step size was $0.10 \mathrm{log}$ units. Thresholds were measured dynamically after onset of the $3.6 \mathrm{log} \mathrm{td}$ background, which abruptly replaced the $1.6 \mathrm{log}$ td background. The threshold averaged from 20-30 seconds after background onset was computed using interpolation methods described previously. ${ }^{15}$

\section{Short wavelength automated perimetry (SWAP)}

After subjects rested for at least 5 minutes, SWAP testing commenced using the Humphrey field analyser (HFA) (Zeiss Humphrey, Dublin, CA, USA). (Generally, 30-2 visual fields were obtained using an HFA II, and 24-2 visual fields were obtained using an HFA I.) Standard clinical procedures were used for size $\mathrm{V}\left(1.7^{\circ}\right.$ diameter $)$ tests presented using the full threshold program. The eye not used for foveal psychophysical testing was assessed first. In this paper, "eye 1 " refers to the first eye tested using SWAP, and "eye 2" refers to the second eye. All mean deviations (MDs) reported here are for 24-2 fields, whether measured directly or reduced from 30-2 data. Age corrected sensitivities were computed for each point in the 24-2 visual field and were used to derive age corrected sensitivities for various portions of the visual field, just as the MD represents age corrected sensitivity for the entire field. Sensitivities were derived for each of four rings, defined by the most eccentric $\mathrm{x}$ or $\mathrm{y}$ coordinate positions within these rings: $3^{\circ}, 9^{\circ}, 15^{\circ}$, and $21^{\circ}$, respectively. The positions in the visual field that corresponded to the blind spot and to its mirror image across the vertical meridian were omitted from the calculations involving only portions of the 24-2 visual field. The points at $27^{\circ}$ eccentricity were omitted also. Division of the SWAP visual field into concentric regions for non-glaucomatous conditions has been made previously. ${ }^{25}$ Age corrected norms and the factors used to make the age corrections were provided by Chris Johnson (personal communication), based on data from 348 subjects. ${ }^{27}$

\section{Frequency doubling perimetry (FDP)}

After subjects had an opportunity to rest, FDP was administered using the full threshold program on the Humphrey FDT visual field instrument (software version 2.60; Zeiss Humphrey, Dublin CA; Welch Allyn, Skaneateles Falls, NY, USA). Administration of FDP departed from the standard clinical procedure in one way: the right eye was not necessarily assessed first. Instead, the eye not used for foveal psychophysical testing was assessed first. Corrections were made for the FDP program's use of different norms for right and left eyes. ${ }^{28}$ The precise correction factor was provided by Chris Johnson (personal communication). Once it became apparent that there were no substantial training effects, the right eye was tested first. 
Assessment of the retina and optic nerve head Fundus and optic nerve head stereo colour fundus photographs were obtained using standard clinical procedures, including dilation and cycloplegia. The photographs were examined by a glaucoma specialist (JRS) masked from all other data. Each eye was graded "S" ( some level of suspicion for glaucoma, however small), "G" (glaucoma), "P" (other pathology), or " $\mathrm{N}$ " (normal). Subjects were excluded from the study if either eye received a score other than "N." Small, non-confluent macular drusen or a comparable level of macular pigmentary change were not grounds for exclusion. Photographs were taken at initial testing sessions only. There was no evidence of tamoxifen retinopathy in any subject.

\section{Statistical analyses}

All $p$ values are for two sided tests. Statistical analyses were conducted using SYSTAT 8.0 (Richmond, CA, USA).

\section{RESULTS}

\section{Visual field sensitivities and the duration of tamoxifen} use

The SWAP MDs from subjects' first or only testing sessions are given in figure 1 for each eye as a function of the duration of tamoxifen use. The horizontal broken lines represent the medians of the respective MDs, and the vertical broken lines represent the median duration of tamoxifen use. For each eye, the SWAP MDs were distributed non-randomly across the four quadrants defined by these medians. $(p=0.014$ for eye $1, p=0.033$ for eye 2; Fisher's exact test of the frequencies in a $2 \times 2$ table). Relatively high MDs were most likely to occur for subjects who used tamoxifen for comparatively short periods, whereas relatively low SWAP MDs were most likely to occur for subjects who used tamoxifen for comparatively long periods.

As expected, ${ }^{29}$ the SWAP and FDP MDs were mutually correlated $(r=0.49$ for eye 1 and $r=0.62$ for eye $2 ; \mathrm{p}<0.001$ for each eye). However, the FDP MDs appeared to be related little or not at all to the duration of tamoxifen use $(p=1.00$ for each eye, Fisher's exact test). This negative result indicates that the duration dependence of the SWAP MDs was not representative for visual fields generally. (For each eye, the FDP scores averaged about $-2 \mathrm{~dB}$, but these averages did not differ significantly from the $-1.5 \mathrm{~dB}$ averages obtained for 32 non-tamoxifen control subjects.)

To compare the SWAP and FDP results directly, it was necessary to provide a common scale for comparison, since a between subject difference of $1 \mathrm{~dB}$ of SWAP MD corresponded to a between subject difference of about $0.34 \mathrm{~B}$ of FDP MD (that is, 0.34 was the average slope for the two linear regression equations, one per eye, describing the FDP MD as a function of the SWAP MD). To put the two types of MDs on a common scale, the SWAP and FDP MDs were standardised for each eye (that is, the MDs were converted to $\mathrm{z}$ scores). The differences between the standardised SWAP and FDP MDs then were assessed for randomness in the same manner that the SWAP MDs themselves were assessed. The distributions of the standardised MD differences were significantly different from random $(p=0.015$ for eye 1 , $\mathrm{p}=0.015$ for eye 2; Fisher's exact test of the frequencies in a $2 \times 2$ table). The standardised MD differences are shown in figure 2 .

All statistics concerning analyses of $2 \times 2$ tables were computed after omitting data from the four subjects who reported using tamoxifen for 2 years-that is, for the median duration of use. However, the conclusions would have been the same had all four subjects been assigned to the above 2 year group or the below 2 year group.

The dependence of SWAP sensitivities on the duration of tamoxifen use was most evident in the visual field periphery.
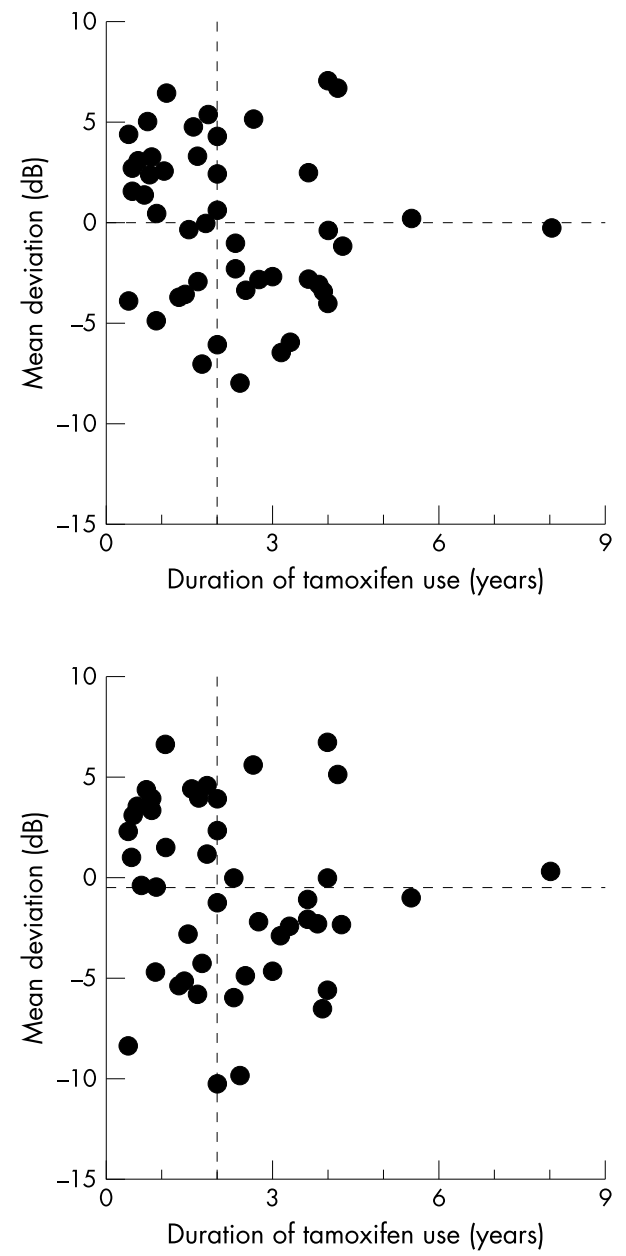

Figure 1 SWAP mean deviations (MDs) from subjects' first or only testing sessions are plotted for eye 1 (top) and eye 2 (bottom). The horizontal broken lines represent the medians of the MDs, and the vertical broken lines represent the median duration of tamoxifen use, which was 2 years. For reference, the lower $5 \%$ limit of normal is -5.39 $\mathrm{dB}$ and the upper $95 \%$ percentile is $4.94 \mathrm{~dB}$.

Figure 3 plots the age corrected SWAP sensitivities for each of four rings of progressively greater retinal eccentricity. Data from the two eyes are shown together. The patterns for the two eyes were similar.

The sensitivities for the outermost ring separated into two apparent clusters: one for subjects with generally high sensitivities and often short histories of tamoxifen use, and the other for subjects with generally low sensitivities and often long histories of tamoxifen use. The reduction of sensitivity with increasing eccentricity tended to be greatest for those subjects in the lower cluster who used tamoxifen for relatively short durations. Thus, for subjects in the low sensitivity cluster, the reduction of sensitivity from the second to the third ring decreased with the duration of tamoxifen use $(r=-0.47$ for eye $\mathrm{l}$ and $r=-0.57$ for eye 2; nominal p values 0.021 and 0.004 , respectively). For subjects in the high sensitivity cluster, there was little or no change with duration $(r=-0.13$ and $r=0.11)$. The separation into these two clusters is essentially what precluded the MD data (figs 1 and 2) from being analysed using linear regression.

To prevent the data from atypically long duration of use subjects from influencing the statistics disproportionately, all correlations were computed after data were excluded from the two subjects using tamoxifen for longer than 5 years. The 

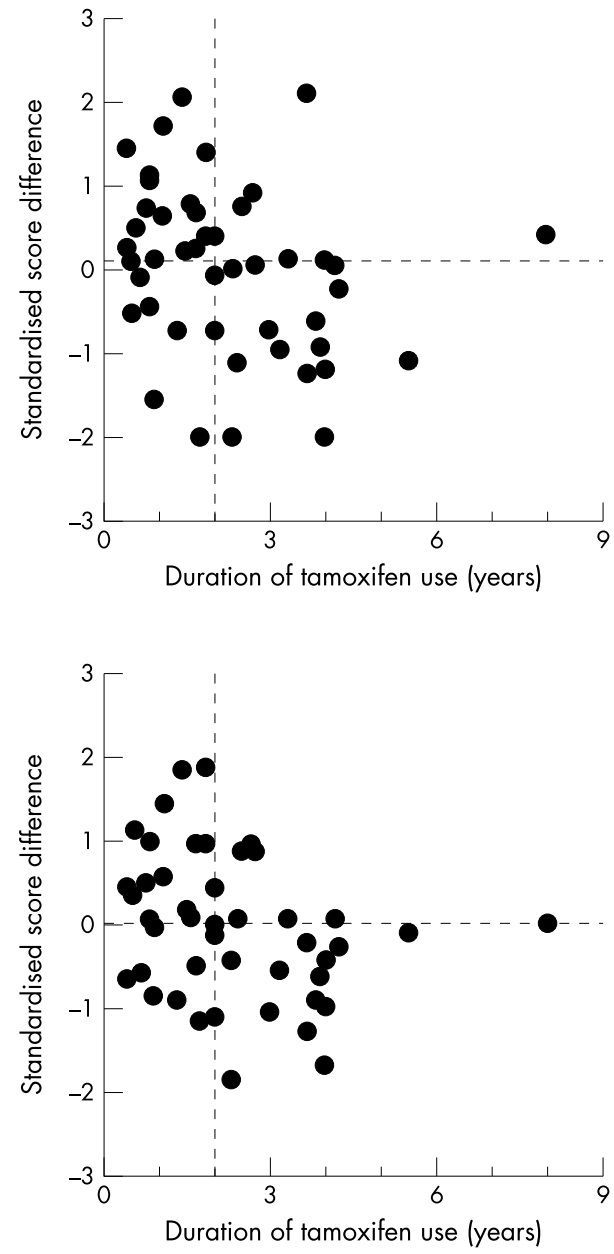

Figure 2 The standardised SWAP MDs minus the standardised FDP MDs for eye 1 (top) and eye 2 (bottom).

patterns evident in figure 3 indicate that tamoxifen can affect SWAP visual fields by affecting the sensitivity decline that occurs with increasing eccentricity.

\section{Foveal SWS cone mediated sensitivities}

On theroretical grounds, ${ }^{21}$ the SWAP sensitivities in the visual field periphery were expected to depend on at least three factors involving SWS cone mediated sensitivity: (1) the rate of sensitivity decline with increasing eccentricity, (2) the baseline sensitivity level-that is, the sensitivity level at yellow background illuminances too dim to induce appreciable sensitivity loss, and (3) an adaptation factor-that is, the degree of desensitisation induced by the yellow background for SWAP. For modelling purposes, the first of these three factors was represented by the reduction of SWAP sensitivity from the second to the third ring. Estimates for the last two factors were derived from the foveal psychophysical data.

The baseline sensitivity factor was represented by the SWS cone mediated sensitivity for the dimmest yellow foveal background ( $1.6 \log \mathrm{td})$. The adaptation factor was represented by the elevation of SWS cone mediated thresholds from the dimmest yellow foveal background ( $1.6 \mathrm{log} \mathrm{td})$ to the brightest ( $3.6 \log \mathrm{td}$ ). For reference, the SWAP background luminance is $2.0 \mathrm{log} \mathrm{cd} / \mathrm{m}^{2},{ }^{10}{ }^{11}$ which corresponds to about $3.0 \log$ td for a typical pupil size.

The SWAP sensitivities in the outermost ring were modelled as a multilinear combination of the aforementioned three factors. This three factor model accounted for $66 \%$ of the variance of the SWAP sensitivities in the outermost ring $(\mathrm{R}=0.81, \mathrm{n}=34)$. All three factors were significant $(\mathrm{p}<0.001$ for the "eccentricity factor", $\mathrm{p}<0.001$ for the "baseline sensitivity factor", and $\mathrm{p}=0.023$ for the "adaptation factor"). The fit

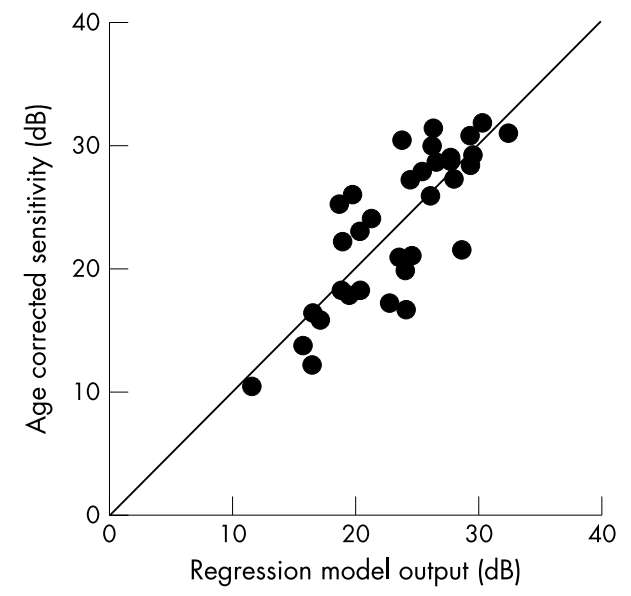

Figure 4 Fit of the three factor multilinear regression model to the age corrected SWAP sensitivities in the outermost ring. The three factors were: (1) an eccentricity factor (age corrected sensitivity for SWAP ring 2 minus age corrected sensitivity for SWAP ring 3), (2) a baseline sensitivity factor (log sensitivity for a 1.6 log td foveal background), and (3) an adaptation factor (log threshold for a $3.6 \mathrm{log}$ td $580 \mathrm{~nm}$ foveal background minus log threshold for a $1.6 \log$ td $580 \mathrm{~nm}$ foveal background).
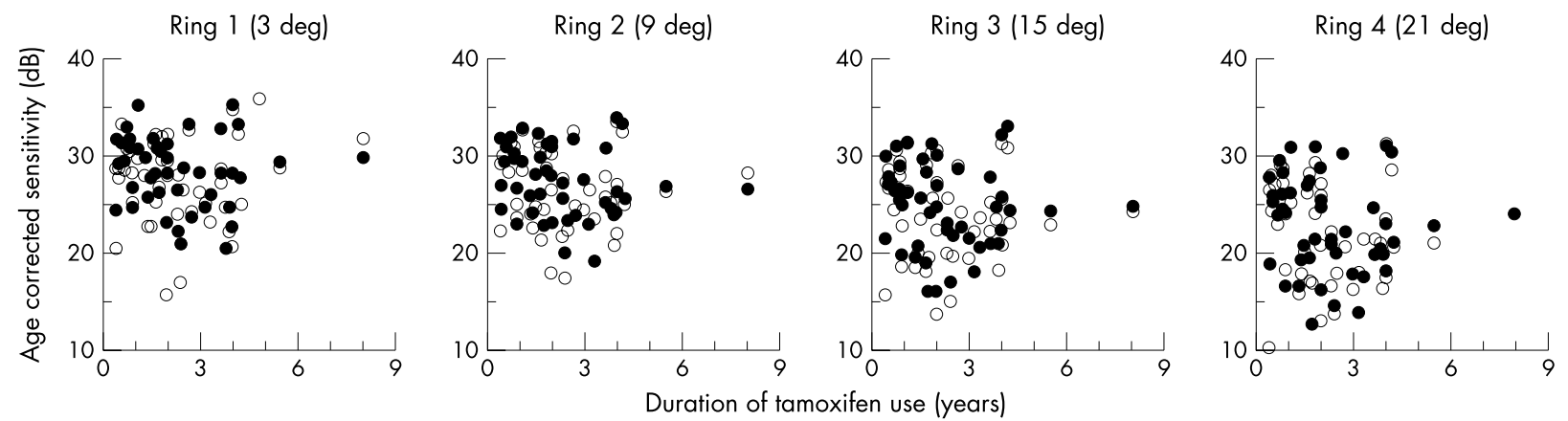

Figure 3 Age corrected sensitivities derived from the SWAP scores for each of four rings of progressively greater retinal eccentricity. Each ring was defined by the most eccentric $x$ or $y$ coordinate position within that ring: $3^{\circ}, 9^{\circ}, 15^{\circ}$, and $21^{\circ}$, respectively. Filled symbols represent data from eye 1 , and open symbols, data from eye 2. (For computational purposes, the two clusters of sensitivities in the outermost ring may be regarded as lying on opposite sides of a line corresponding to the equation $y=1.9 x+20$, where $y$ represents age corrected sensitivity and $x$ represents the duration of tamoxifen use.) 
of the model to the data is given in figure 4 . The relative weights of the three factors (that is, the standardised regression coefficients) were $-0.55,0.51$, and -0.26 , respectively, meaning that the foveally derived estimate of the adaptation factor was about half as important as each of the other two factors. The sensitivity factor did not appear to depend on the duration of tamoxifen use, even after allowing for age effects. The relation of the adaptation factor to the duration of tamoxifen use is shown in figure 5 . There is some suggestion of a change in the adaptation factor at about 2 years, but any effect of duration was not significant.

The adaptation factor measured the ability of yellow backgrounds to affect SWS cone mediated sensitivity in the steady state. There was evidence that tamoxifen affected the dynamics of visual adaptation in SWS cone pathways. The degree of desensitisation shortly after onset of a bright yellow background ( $3.6 \log \mathrm{td}, 580 \mathrm{~nm}$ ) was assessed by comparing thresholds averaged from 20-30 seconds after background onset with thresholds measured after attainment of a steady state level of sensitivity. ${ }^{15}$ The degree of desensitisation at 20 30 seconds was correlated weakly, but significantly, with the duration of tamoxifen use $(\mathrm{r}=0.38, \mathrm{p}=0.028)$.

\section{DISCUSSION}

The effects of tamoxifen on perimetric sensitivities depend on the type of visual field test administered. We found that tamoxifen affected SWAP visual fields, but not FDP visual fields, in a manner related significantly to the duration of tamoxifen use. For SWAP, relatively high MDs were most likely to occur for subjects who used tamoxifen for comparatively short periods, whereas relatively low MDs were most likely to occur for subjects who used tamoxifen for comparatively long periods.

Previously, Gorin et al ${ }^{9}$ reported that white on white visual fields were unaffected by tamoxifen. However, these investigators administered 10-2 rather than $24-2$ or 30-2 visual fields, and they tested women who used tamoxifen for more than 5 years or else had used tamoxifen for about 5 years but then discontinued use. In contrast, almost all subjects in our study were using tamoxifen for less than 5 years. Furthermore, we found the effects of tamoxifen to be most evident in the visual field periphery. Thus, it remains possible that tamoxifen affects white on white visual fields over the course of a 5 year treatment period. Definitive determination requires additional testing, preferably involving comparison of SWAP with white on white perimetry.

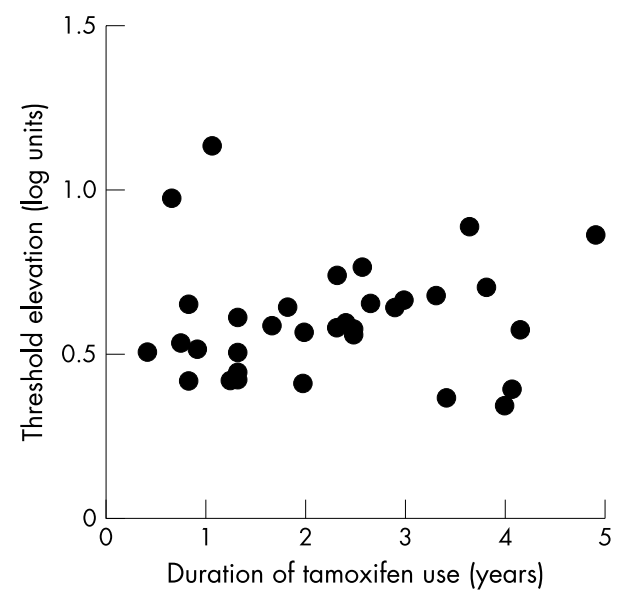

Figure 5 The relation of the adaptation factor to the duration of tamoxifen use. The adaptation factor is defined by the elevation of steady state, SWS cone mediated thresholds from the 1.6 to the $3.6 \mathrm{log}$ td $580 \mathrm{~nm}$ background.
The foveal colour vision changes reported by Gorin et a ${ }^{9}$ were not profound, and the changes in SWAP sensitivities are unlikely to impact a person's ability to function in her environment. However, the changes in SWAP visual fields might affect the utility of SWAP as a means of glaucoma assessment for women who use tamoxifen. The utility of FDP appears less likely to be altered.

There is a general consensus that standard doses of tamoxifen occasionally cause ocular toxicities that are evident on routine ophthalmoscopic or biomicroscopic examination. $^{30}$ Noureddin et $a l^{31}$ found that eight of 65 women using standard doses of tamoxifen developed corneal, retinal, or optic nerve head changes, and, in some cases, two types of change. Noureddin $e t$ al $^{31}$ used a longitudinal design to show that the development of toxicity was related significantly to the duration of tamoxifen use. The absence of observable ocular toxicities in our subjects suggests that biologically based selection factors affected subject recruitment in our cross sectional study, which was restricted to subjects with excellent central vision and no observable ocular pathology. Many of the women whom we tested reported experiencing "dry eye" that they attributed to tamoxifen use, so some subjects may have volunteered for our study because they noticed some visual change.

The results of our study indicate that tamoxifen affects the response of SWS cone pathways, and there was some evidence that tamoxifen affects the adaptation properties of these pathways. If tamoxifen affects the adaptation properties of SWS cone pathways, it probably affects retinal neural function. ${ }^{12}$ Since tamoxifen is a SORM, the results of our study thus provide the first concrete suggestion of a role for retinal ORs. ${ }^{5-8}$

For tamoxifen users, the SWAP sensitivities in the visual field periphery depended on the following three factors: (1) the rate of sensitivity decline with increasing eccentricity, (2) the sensitivity level at yellow background illuminances too dim to induce appreciable sensitivity loss, and (3) the degree of desensitisation induced by a brighter yellow background, such as that employed by SWAP. Because the estimates for the last two factors were derived from foveal data, the effects of tamoxifen on either or both of these factors may be quite strong if measured directly in the visual field periphery. This hypothesis needs to be tested.

Even if the means by which tamoxifen alters SWS cone mediated vision is understood only incompletely, SWAP may potentially provide a method for monitoring the body's changing response to tamoxifen. Evaluating this possibility requires a prospective longitudinal study.

\section{ACKNOWLEDGEMENTS}

This research was supported by NIH grants 5R01 EY12737 and 5P30 CA69533 (Bethesda, MD), and by an unrestricted grant from Research to Prevent Blindness (New York, USA). We thank the Oregon and Southwest Washington affiliate of the Komen Foundation for assistance with subject recruitment, and we thank Chris Johnson, PhD, for providing visual field norms and correction factors.

\section{Authors' affiliations \\ A Eisner, Neurological Sciences Institute, Oregon Health \& Science University, OR, USA \\ A Eisner, J R Samples, Casey Eye Institute, Oregon Health \& Science University, OR, USA \\ D F Austin, Department of Public Health and Preventive Medicine, Oregon Health \& Science University, OR, USA}

\section{REFERENCES}

1 Lonard DM, Smith CL. Molecular perspectives on selective estrogen receptor modulators (SERMs): progress in understanding their tissue-specific agonist and antagonistic actions. Steroids 2002;67:15-24. 
2 Morello KC, Wurz GT, DeGregoriao MW. SERMS: current status and future trends. Crit Rev Oncol Hematol 2002;43:63-76.

3 Osborne CK. Tamoxifen in the treatment of breast cancer. N Engl J Med 1998:339:1609-18.

4 Wolff AC, Davidson NE. Use of SERMs for the adjuvant therapy of early-stage breast cancer. Ann N Y Acad Sci 2001;949:80-8.

5 Munaut $C$, Lambert V, Noel A, et al. Presence of oestrogen receptor type beta in human retina. $\mathrm{Br} J$ Ophthalmol 2001;85:877-82.

6 Wickham LA, Gao J, Toda I, et al. Identification of androgen, estrogen and progesterone receptor mRNAs in the eye. Acta Ophthalmol Scand 2000;78: 146-53.

7 Ogueta SB, Schwartz SD, Yamashita CK, et al. Estrogen receptor in the human eye: influence of gender and age on gene expression. Invest Ophthalmol Vis Sci 1999:40:1906-11.

8 Kobayashi K, Kobayashi H, Ueda M, et al. Estrogen receptor expression in bovine and rat retinas. Invest Ophthalmol Vis Sci 1998;39:2105-10.

9 Gorin MB, Day R, Costantino JP, et al. Long-term tamoxifen citrate use and potential ocular toxicity. Am J Ophthalmol 1998;125:493-501.

10 Sample PA. Short-wavelength automated perimetry: its role in the clinic and for understanding ganglion cell function. Prog Ret Eye Res 2000;19:369-83.

11 Wild JM. Short wavelength automated perimetry. Acta Ophthalmol Scand 2001;79:546-59

12 Dacey DM. Parallel pathways for spectral coding in primate retina. Annu Rev Neurosci 2000;23:743-75.

13 Eisner A. Longitudinal changes of visual function over 18 months: evaluation of eyes with high- and low-risk macular degeneration characteristics. Doc Ophthalmol Proc Ser 1993:56:175-87.

14 Eisner A, Fleming SA, Klein ML, et al. Sensitivities in older eyes with good acuity: cross-sectional norms. Invest Ophthalmol Vis Sci 1987;28:1824-31.

15 Eisner A, Samples JR, Campbell HM, et al. Foveal adaptation abnormalities in early glaucoma. J Opt Soc Am A 1995;12:2318-28.

16 Robertson JA, Farnell Y, Lindahl LS, et al. Estradiol up-regulates estrogen receptor messenger ribonucleic acid in endometrial carcinoma (Ishikawa) cells by stabilizing the message. J Mol Endocrinol 2002;29:125-35.

17 Anderson AJ, Johnson CA. Mechanisms isolated by frequency-doubling technology perimetry. Invest Ophthalmol Vis Sci 2002;43:398-401.
18 Bryant J, Fisher B, Dignam J. Duration of adjuvant tamoxifen therapy. J Natl Cancer Inst Monogr 2001;30:56-61.

19 Clarke R, Leonessa F, Welch JN, et al. Cellular and molecular pharmacology of antiestrogen action and resistance. Pharmacol Rev $2001 ; 53: 25-71$.

20 Bernstein L, Deapen D, Cerhan JR, et al. Tamoxifen therapy for breast cancer and endometrial cancer risk. J Natl Cancer Inst 1999;91:1654-62.

21 Pearson PM, Swanson WH. Chromatic contrast sensitivity: the role of absolute threshold and gain constant in differences between the fovea and the periphery. J Opt Soc Am A 2000;17:232-43.

22 Eisner A, Cioffi GA, Campbell HM, et al. Foveal flicker sensitivity abnormalities in early glaucoma: associations with high blood pressure. J Glaucoma 1994;3(suppl.):S19-S31.

23 Adams AJ, Rodic R, Husted R, et al. Spectral sensitivity and color discrimination changes in glaucoma and glaucoma-suspect patients. Invest Ophthalmol Vis Sci 1982;23:516-24.

24 Eisner A. Multiple components in photopic dark adaptation. J Opt Soc Am A 1986;3:655-66

25 Nomura $R$, Terasaki $\mathrm{H}$, Hirose $\mathrm{H}$, et al. Blue-on-yellow perimetry to evaluate $\mathrm{S}$ cone sensitivity in diabetics. Ophthalmic Res 2000;32:69-72.

26 Remky A, Lichtenberg $K$, Elsner $A E$, et al. Short wavelength automated perimetry in age related maculopathy. Br J Ophthalmol 2001;85:1432-36.

27 Johnson CA, Sample PA, Cioffi GA, et al. Structure and function evaluation (SAFE): I. criteria for glaucomatous visual field loss using standard automated perimetry (SAP) and short wavelength automated perimetry (SWAP). Am J Ophthalmol 2002;134:177-85

28 Adams CW, Bullimore MA, Wall M, et al. Normal aging effects for frequency doubling technology perimetry. Optom Vis Sci 1999;76:582-87.

29 Landers J, Goldberg I, Graham S. A comparison of short wavelength automated perimetry with frequency doubling perimetry for the early detection of visual field loss in ocular hypertension. Clin Exp Ophthalmol 2000;28:248-52

30 Fraunfelder FT, Fraunfelder FW. Drug-induced ocular side effects, 5th ed. Boston: Butterworth Heinemann, 2001:471-5.

31 Noureddin BN, Seoud M, Bashshur Z, et al. Ocular toxicity in low-dose tamoxifen: a prospective study. Eye 1999;13:729-33. 